Please do not remove this page

RMIT

UNIVERSITY

\title{
Sensing unsteady pressure on MAV Wings: a new method for turbulence alleviation
}

Marino, Matthew; Watkins, Simon; Sabatini, Roberto; Gardi, Alessandro Giacomo Maria

https://researchrepository.rmit.edu.au/esploro/outputs/9921862248601341/filesAndLinks?institution=61RMIT_INST\&index=null

Marino, M., Watkins, S., Sabatini, R., \& Gardi, A. G. M. (2014). Sensing unsteady pressure on MAV Wings: a new method for turbulence alleviation. Applied Mechanics and Materials, 629, 48-54.

https://doi.org/10.4028/www.scientific.net/AMM.629.48

Document Version: Accepted Manuscript

Published Version: https://doi.org/10.4028/www.scientific.net/AMM.629.48

Repository homepage: https://researchrepository.rmit.edu.au

(C) 2014 Applied Mechanics and Materials

Downloaded On 2023/04/26 22:20:30 +1000

Please do not remove this page 
Thank you for downloading this document from the RMIT Research Repository.

The RMIT Research Repository is an open access database showcasing the research outputs of RMIT University researchers.

RMIT Research Repository:http://researchbank.rmit.edu.au/

\section{Citation:}

Marino, M, Watkins, S, Sabatini, R and Gardi, A 2014, 'Sensing unsteady pressure on MAV Wings: a new method for turbulence alleviation', Applied Mechanics and Materials, vol. 629, pp. 48-54.

See this record in the RMIT Research Repository at:

https://researchbank.rmit.edu.au/view/rmit:24971

Version: Accepted Manuscript

Copyright Statement: (c) 2014 Applied Mechanics and Materials

Link to Published Version:

http://dx.doi.org/10.4028/www.scientific.net/AMM.629.48 


\title{
Sensing Unsteady Pressure on MAV Wings: a New Method for Turbulence Alleviation
}

\section{Matthew James Marino ${ }^{1, a^{*}}$, Simon Watkins ${ }^{1, b}$, Roberto Sabatini ${ }^{1, c}$ and Alessandro Gardi ${ }^{1}$}

\author{
${ }^{1}$ School of Aerospace, Mechanical and Manufacturing Engineering, \\ RMIT University, Melbourne, VIC 3000, Australia \\ amatthew.marino@rmit.edu.au, ${ }^{b}$ simon.watkins@rmit.edu.au, ${ }^{c}$ roberto.sabatini@rmit.edu.au
}

\begin{abstract}
Keywords: Micro Air Vehicle (MAV), Pressure Sensing, Turbulence, Unsteady Pressures, Unmanned Aerial Systems (UAS).
\end{abstract}

\begin{abstract}
Experiments at low Reynolds numbers were performed on a pressure tapped NACA2313 wing in a $3 \times 2 \times 9$ meter wind tunnel under nominally smooth $(\mathrm{Ti}=1.2 \%)$ and turbulent $(\mathrm{Ti}=$ $7.2 \%)$ flows at a mean flow velocity of $8 \mathrm{~ms}^{-1}(\mathrm{Re} \approx 120,000)$. The NACA2313 wing is a replica of a Micro Air Vehicle (MAV) wing of the Flash 3D aircraft used at RMIT University for research purposes. Unsteady surface pressures were measured to understand if the information could be adopted for resolving turbulence-induced perturbations and to furthermore use it in a turbulence mitigation system. Two span-wise locations of chord-wise pressure were acquired when tested under the two different flow conditions. It was discovered that at both span-wise locations, a local Coefficient of Pressure $(\mathrm{Cp})$ held high correlation to the chord-wise $\mathrm{Cp}$ integration and allowed for a linear relationship to be formed between the two variables. The defined relationship provided a $95 \%$ confidence for angles of attack below stall and was used to estimate the integrated cord-wise pressure coefficient at a particular span wise location. The relationship between a single pressure tap and the integrated $\mathrm{Cp}$ of that chord-wise section was valid for the two different span-wise locations with similar defining equations. As one pressure tap is sufficient to adequately estimate the integrated $\mathrm{Cp}$ on a chord-wise wing section, a limited amount of pressure taps across the wings span approximates the pressure distribution across the span and eventually approximates the flight perturbations. Being a novel method of sensing aircraft disturbance, applications are not restricted to MAV. The methodology presented could very well be applied to larger aircraft to reduce the effects of turbulence within the terminal area and can provide other means of active stabilization.
\end{abstract}

\section{Introduction}

Micro Air Vehicles (MAVs) are small, light-weight aircraft, which are remotely or autonomously controlled with flight endurance of up to 30 minutes depending on their design and purpose [1]. Their capability to survey an area without the use of human ground personnel has demonstrated cost and risk benefits when line of sight reconnaissance is not possible [1]. MAVs would typically be launched by an operator in the field, fly short distances either unassisted or with low levels of human control at low altitude into complex terrain, then loiter or perch for data collection. As atmospheric winds increase, the complexity of holding relatively stable and steady flight increases and under even moderate winds the flight of an MAV may become violent and perhaps uncontrollable due to turbulence in the Atmospheric Boundary Layer (ABL) [2]. In complex terrains, the frictional effects of buildings and vegetation lead to a highly turbulent velocity profile within the ABL, which can extend as high as $1000 \mathrm{~m}$ in high-rise city locations [3]. Under low atmospheric wind speeds $(<\sim 5 \mathrm{~m} / \mathrm{s})$ turbulence is commonly created via thermal effects, whereas under higher wind speeds $(\sim 5 \mathrm{~m} / \mathrm{s})$ mechanical mixing dominates the turbulence generation mechanisms [4]. Successful MAV missions require a stable platform for acquisition of relatively stable video or other data [1]. Human-in-the-loop methods of control have shown to be slow to respond to flight perturbations due to the time involved with a human observing a flight deviation, processing the information and then reacting with a suitable control command leading to 
a flight correction [5]. In piloted flight trials of MAVs, where aspects of the relative atmospheric turbulence characteristics were replicated within a large wind tunnel, an experienced pilot attempted to hold straight and level flight, the workload was shown to be high [6]. Under the highest level of replicated turbulence intensity $(13.3 \%)$, the fixed wing MAVs frequently experienced rapid departures from controlled flight and this often resulted in terminal departures. Such issues have led to research in MAV flight stabilization with application of passive and active control methods [6]. In an attempt to learn from nature's flyers, inspiration has been sought from insects and birds as they frequently fly in severe levels of turbulence within cities without incident [7]. It seems likely that nature's flyers "feel" the surrounding air, enabling a rapid response to maintain a steady flight state, perhaps in a similar way to humans maintaining a steady vertical stance when moving over rough ground. Engineering attempts have been made capture the unsteady nature of turbulence around wings and how turbulence significantly influences wing performance and local flow structures [8]. Other biomimetic inspirations include wing morphing and flexible wings [9]. Flexible wings have shown promise in damping the effects of ABL turbulence, however total turbulence mitigation was not possible [10]. Active control systems utilizing Inertial Measurement Units (IMU) have also shown promising results with significant reductions in response time to turbulence perturbation in comparison to basic human control response [6]. The methods of active control to detect perturbations involving IMU systems are unsuitable due to the phase lag between disturbance and control response. The lag is detrimental to achieving steady level flight under turbulent free stream conditions. From pilot experience and IMU data, counteracting the roll inputs from turbulence appears to be the main challenge that needs to be overcome for fixed wing MAVs [6]. Although active control methods have been well researched, IMU have been the primary sensors to feedback aircraft response and little research has been perfume on considering alternative methods of sensing disturbances. Sensing and measuring the flow directly over the aircraft wings may offer the possibility in acquiring flow data directly and further to use this information in a turbulence mitigation system. Furthermore the methodology presented in this paper allows for measurement of turbulence rather than aircraft response and may prove to be beneficial in effective and active control.

\section{Experimental Setup}

The NACA2313 wing was constructed from lightweight "blue" foam and pressure tapping "modules" were manufactured using rapid prototyping methods allowing Polyvinyl Chloride (PVC) tube connections to a digital pressure measuring system. The NACA2313 wing embodied four span-wise rapid prototyping modules that enabled pressures to be acquired at multiple locations along the chord. The inner and outer modules feature $125 \mathrm{~mm}$ and $375 \mathrm{~mm}$ respectively from the wings centreline. A rigid central support held the wing at the mid-span, 5 chord lengths above the tunnel floor. Clearance of over four chord lengths between the wing tips and wind tunnel wall ensured the flow over the wing was not influenced by the walls boundary layer. Fig. 1 illustrates a schematic of the module and the NACA2313 test aerofoil with all pressure modules installed.

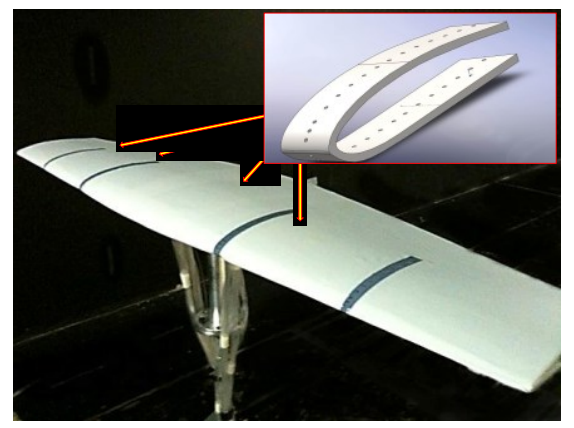

Fig. 1. Schematic of the module. 
The tunnel blockage was less than $1 \%$ and was concluded that its influence was insignificant when acquiring surface pressures. The upper and lower surfaces of the NACA2313 wing were pressure-tapped at the same chord-wise points. The distance between each tap near the leading edge was small to ensure data resolution at the critical lifting region of the chord-wise pressure strip. Pressure-tap separation increased as $\mathrm{x} / \mathrm{c}$ increased. The results presented in this paper were obtained from the data taken from pressure modules on the right side of the wing. Each $2 \mathrm{~mm}$ diameter hole, on each pressure module, interfaced with the outer $2 \mathrm{~mm}$ diameter of each PVC tube, which were glued in place with Epoxy, cut and then sanded flush to the wings surface to follow the curvature of the wing. The other end of each PVC tube was connected to a dynamic pressure measurement system from Turbulent Flow Instrumentation (TFI). The PVC tubes were $1600 \mathrm{~mm}$ long with an internal diameter of $1 \mathrm{~mm}$ and as such each tap shared a common dynamic response. The response dynamic response of the tubing system was corrected to a flat frequency response up to $150 \mathrm{~Hz}$ using the Berge and Tijdemen tube correction method [11]. This tube correction method has been successful in rectifying the non-linear tube response of a hollow tube with constant internal diameter at various lengths [12]. The calibration also takes into account the effects of the surrounding environment and several other factors.

\section{Measurement of Fluctuating Pressures}

The transient and mean Pressure Coefficients $\left(C_{P}\right)$ are given as:

$$
\begin{aligned}
& C_{p}{ }^{\prime}=\frac{P^{\prime}-P_{\infty}}{1 / 2 \rho V^{2}} \\
& C_{p}=\frac{P-P_{\infty}}{1 / 2 \rho V^{2}}
\end{aligned}
$$

where $C p^{\prime}$ and $C p$ are the transient and mean pressure coefficients respectively, $P^{\prime}$ and $P$ are the transient and mean local pressures respectively, $P_{\infty}$ is the ambient static pressure, $\rho$ is density of air and $V$ is the mean stream-wise velocity. $P_{\infty}$ was estimated by acquiring time-averaged pressures from the static port of a Pitot-Static tube placed at the location of aerofoil testing. Each test was performed at a data acquisition rate of $2000 \mathrm{~Hz}$ at a sample time of 3 minutes. A digital low-pass Butterworth filter, with a cut off frequency of $150 \mathrm{~Hz}$, was applied post processing to eliminate noise and any data aliasing within the system.

\section{Wind Tunnel Test and Flow Properties}

All experiments were conducted in the "RMIT University Industrial Wind Tunnel". The tunnel is a closed loop return cycle wind tunnel with a $3 \times 2 \times 9 \mathrm{~m}$ test section. In its nominally smooth flow configuration the turbulence intensity at the aerofoil test location was $1.2 \%$ [13]. Whilst this turbulence level is higher than conventional aeronautical tunnels, it must be emphasised that MAVs flying under typical outdoor conditions will generally experience significantly higher levels of turbulence [3]. The tunnel also has a very low acoustic signature as it is fitted with anechoic turning vanes [14]. For tests under elevated turbulence levels, a planar rectangular grid was installed upstream of the contraction of the wind tunnel. The square sections allowing passage of flow where $600 \times 600 \mathrm{~mm}$ with $300 \mathrm{~mm}$ separation and produced a flat time-averaged profile in the vicinity of the test locations however with 7.3\% Turbulence Intensity (Ti) and $0.23 \mathrm{~m}$ longitudinal integral length scale (Lxx). Both flat plat and NACA wing experiments where conducted 8 meters downstream of the grids to allow sufficient mixing for the development of homogeneous free stream turbulence [4]. Whilst the relative turbulence intensity can vary widely depending upon atmospheric wind and MAV speeds and terrain, $7 \%$ turbulence intensity is a value thought to be close to that experienced while flying between $5-10 \mathrm{~m} / \mathrm{s}$ through complex terrain under average atmospheric wind conditions [3]. The schematic of the experiment is illustrated in Fig. 2. 


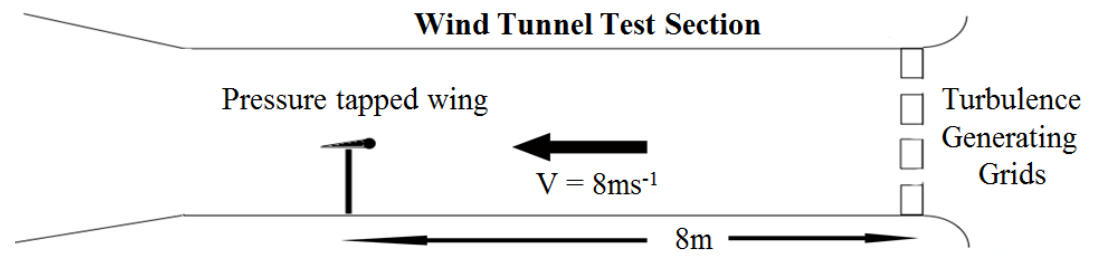

Fig. 2. Schematic of the experiment with tunnel configured to create high turbulence intensity.

\section{The Relationship between a Single Pressure Tap and Integrated $C p$}

A covariance algorithm was used to quantifiably measure the relationship strength between the pressures measured at a single pressure tap and the overall integrated $C p$ over the wing as illustrated in Fig. 3.

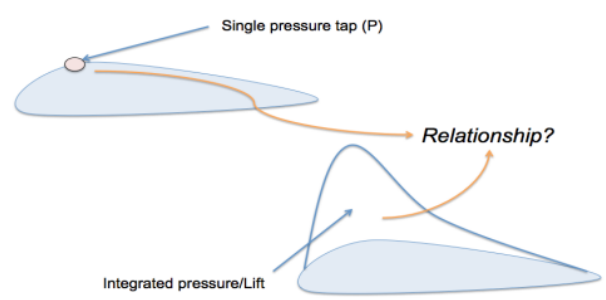

Fig. 3. Correlation between a local and integrated $C p$ for an arbitrary span-wise location.

Due to the structural challenges of tapping pressure in the entire chord, only $49 \%$ of the chord length was integrated. It was found that the partial tapping does not significantly effect the overall correlation between local and integrated $C p$ as the majority (approx. 76\%) of lift and pressure fluctuation occur within this area [15].

$$
r_{C_{p^{\prime}} C_{p}}(m)=E\left\{\left(C_{p, n+m}-\mu_{C_{p}}\right)\left(C_{p, n}{ }^{\prime}-\mu_{C_{p^{\prime}}}\right)^{*}\right\}
$$

where $r_{C_{p^{\prime}} C_{p}}$ is the covariance of variables $C_{p}{ }^{\prime}$ and $C_{p}$ at length $\mathrm{m}, \mu_{C_{p}}$ and $\mu_{C_{p}}$ are the mean values of the two stationary random processes, * denotes the complex conjugate, and $E\{\}$ is the expected value operator [16]. The correlations between each chord-wise tap and the integrated $C p$ over the 3minute sample for inboard and outboard pressure modules is illustrated in Fig. 4.
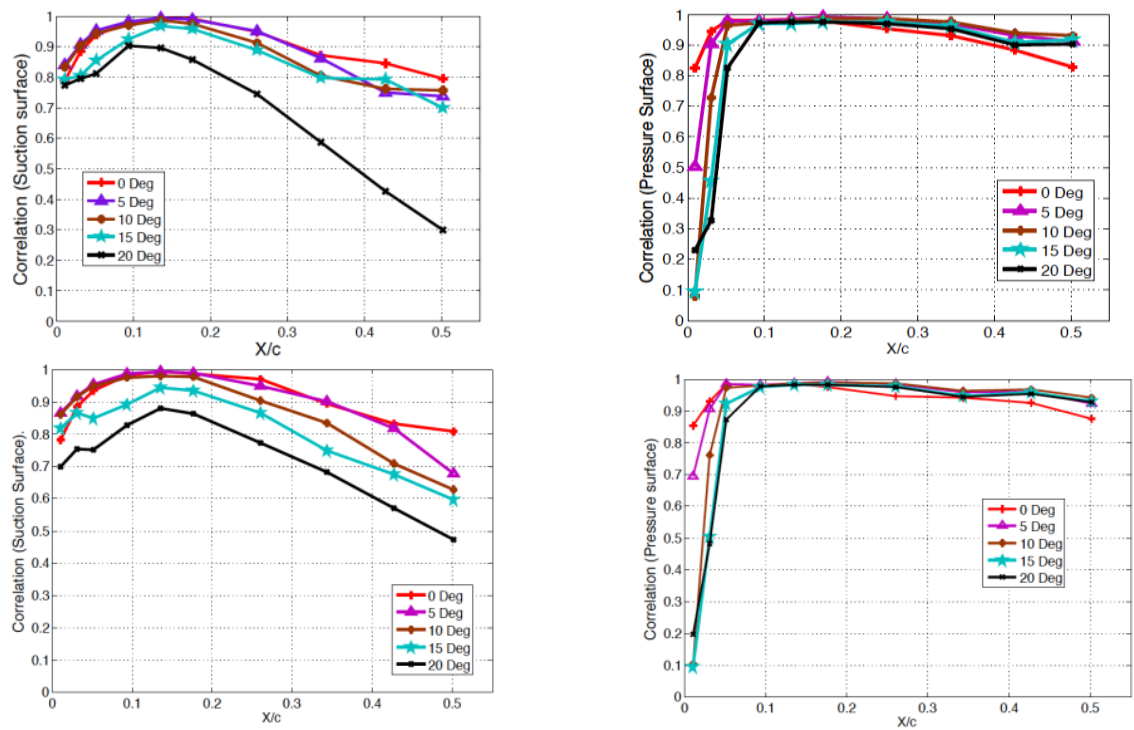

Fig. 4. Correlation between single pressure tap and integrated pressure for suction and pressure surface of the wing for the inboard (top) and outboard (bottom) pressure module in turbulent flow.

$$
(\mathrm{Ti}=7.2 \%, \mathrm{Lxx}=0.21 \mathrm{~m})
$$


The suction surface of the wing displayed correlation above $80 \%$ between $1 \%$ and $20 \% x / c$ for all Angles of Attack (AOA) for the inboard module. The outboard module displayed similar results but demonstrated reduced correlation at 20 degrees AOA. This abnormality was evident and assumed to be the cause of the wing tip vortices impinging on the flow. Both inboard and outboard correlation studies suggested a location of correlation maxima at $14 \% x / c$ across all the values of AOA tested. It can be concluded that the relationship between the pressure tap at $14 \%$ and integrated $C p$ is linear and can be bound to a $95 \%$ confidence if local $C p$ was used to approximate integrated $C p$. The correlation between single pressure taps and integrated $C p$ for the pressure surface of the wing displayed similar results for both inboard and outboard modules. The scatter plot of integrated $C p$ and the local $C p$ for the suction and pressure surface in turbulent flow is illustrated in Fig. 5. The section of chord between 1 and $5 \% x / c$ displayed relatively lower correlation coefficients due to the stagnation point compared to other chord locations. The stagnation point is commonly known to have a constant value of $C p=1$ at arbitrary velocities and is not persuaded by unsteady flow and thus greatly effects the correlation between the pressure taps in this region and integrated $C p$.
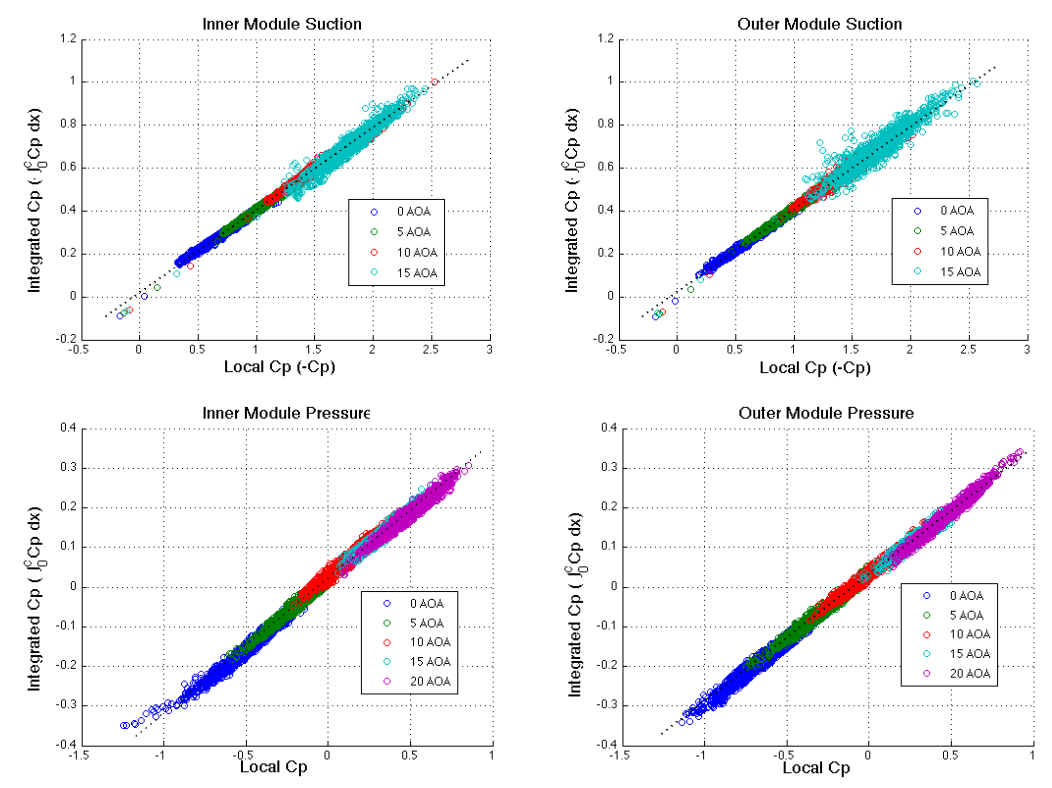

Fig. 5. Scatter plot of integrated $C p$ vs local $C p$ for the suction (top) and pressure (bottom) surface in turbulent flow of the pressure tap at $14 \%$ chord. $(T i=7.2 \%, L x x=0.21 \mathrm{~m}$ )

\section{Approximating Lift from Span-Wise Pressure Taps}

When the AOA, $\alpha$ is less than 5 degrees, lift is calculated by integrating the pressure coefficient over the suction and pressure surface of the wing. For larger AOA, the cosine rule is applied and is given by:

$$
N_{i} \propto \cos \alpha \int C p_{i} d x
$$

where $N$ is the normal force vector per unit span $i$ and $C p_{i}$ are pressure coefficients across the chord. Due to considerably high correlations between the pressure tap at $14 \% x / c$ and integrated $C p$, proportionality between a local $C p$ and integrated $C p$ is modelled and expressed as:

$$
\begin{aligned}
& \int C_{P_{i}} d x \propto C_{\text {Plocal }, i} \\
& \int C_{P_{i}} d x=K C_{\text {Plocal }, i}+C
\end{aligned}
$$

To resolve for lift (for both suction and pressure surfaces of the wing), cosine is introduced as:

$$
N_{i}=\left(K C_{\text {Plocal }, i}+C\right) \cos \alpha
$$


The resolution of lift at each span-wise section is given as:

$$
L_{i}=N_{\text {Suction }, i}+N_{\text {Pressure }, i}
$$

\section{Resolving for Turbulence Induced Roll}

Although MAVs are perturbed in low-atmospheric flight in 6-degrees-of-freedom, it has been found that MAV are more significantly perturbed in roll and greatly effect the capability of acquiring sensory data [17, 18]. Resolving lift at different span-wise points across the wingspan allows for performing measurements of a Turbulence Induced Roll (TIR). TIR is measured by resolving the moments around the aircraft centreline using:

$$
l_{T I R}=x_{1} \frac{b}{2 n}\left(L_{L 1}-L_{R 1}\right)+x_{2} \frac{b}{2 n}\left(L_{L 2}-L_{R 2}\right) \ldots \ldots+x_{n} \frac{b}{2 n}\left(L_{L n}-L_{R n}\right)
$$

where $l_{T I R}$ is the turbulence induced rolling moment, $x$ is the lateral distance between the aircraft centreline and the pressure module, $b$ is the wingspan, $L$ is the lift per span-wise lifting segment at location $n$ on the left and right sides of the wing. The relation between TIR and total aircraft rolling moment is given by:

$$
C_{l_{T}}=C_{l_{A}}+C_{l_{T I R}}
$$

where $C_{l_{T}}$ is the total aircraft rolling moment, $C_{l_{A}}$ is the aircraft rolling moment due to control and $C_{l_{T I R}}$ is the turbulence induced rolling moment. Furthermore the TIR moment is introduced in the lateral equations of motion expressed as:

$$
C_{l_{T}}=C_{l_{\beta}} \beta+C_{l_{p}} \frac{p b}{2 V}+C_{l_{r}} \frac{r b}{2 V}+C_{l_{\delta_{r}}} \delta_{r}+C_{l_{\delta a}} \delta_{a}+C_{l_{T I R}} l_{T I R}
$$

where $\mathbf{C}_{\mathbf{l}_{\boldsymbol{\beta}}}$ is the aircraft rolling moment coefficient due to sideslip $\boldsymbol{\beta}, \mathbf{C}_{\mathbf{l}_{\mathbf{p}}}$ is the roll damping coefficient due to roll rate $p, \mathbf{C}_{\mathbf{l}_{\mathbf{r}}}$ is the roll moment coefficient due to rudder deflection $\boldsymbol{\delta}_{\mathbf{r}}$, and $\mathbf{C}_{\mathbf{l}_{\mathbf{a}}}$ is the roll moment coefficient due to rudder deflection $\boldsymbol{\delta}_{\mathbf{a}}$.

\section{Conclusions and Future Work}

This paper demonstrated the methodology in which a strip of pressure taps across the span of the wing, at an optimum chord-wise location, approximates lifting sections of the wing using a limited amount of pressure taps. The measurements allow the calculation of TIR and offer perturbation information, which can be supplied to the autopilot system, which in term might be a viable and advantageous alternative to current active stability systems using IMU sensors. The applications of this system are not limited to MAVs and can be applied to larger aircraft. It also offers a new means of turbulence correction as this theorised system has the capability of directly sensing turbulence through acquiring unsteady pressure and may prove to be advantageous over systems employing IMU sensors. The future research would focus on the hardware development of the system and test its effectiveness under a low atmospheric flight mission. The idea of feeling the ambient air may well be a new alternative method of mitigating the effects of turbulence for both manned and unmanned aircraft.

\section{References}

[1] J. William R. Davis and B. B. Kosicki, Micro Air Vehicles for Optical Surveillance, The Lincoln Laboratory Journal, vol. 9, pp. 197-213. (1996)

[2] J.M. McMichael and C.M.S. Francis, Micro Air Vehicles - Toward a New Dimension in Flight, DARPA, USA. (1997)

[3] S. Watkins, J. Millbank and B. Loxton, Atmospheric Winds and their Effects on Micro Air Vehicles," AIAA Journal of Aircraft, vol. 44. (2006) DOI: 10.2514/1.22670 
[4] J. Millbank, B. Loxton, S. Watkins and W.H. Melbourne, Replication of Atmospheric Conditions for the Purpose of Testing MAVs, Technical Report, Royal Melbourne Institute of Technology, Melbourne, Australia. (2005)

[5] M. Abdulrahim, Dynamic Characteristics of Morphing Micro Air Vehicles, Master's Thesis, University of Florida, USA. (2004)

[6] M. Abdulrahim, S. Watkins, R. Segal, M. Marino and J. Sheridan, Dynamic Sensitivity to Atmospheric Turbulence of Unmanned Air Vehicles with Varying Configuration, Journal of aircraft, vol. 47, pp. 1873 - 1883. (2010) DOI: 10.2514/1.46860

[7] S. Dhawan, "Bird flight," Sadhana, vol. 16, pp. 275-352. (1991)

[8] M. Marino, S. Ravi and S. Watkins, Optimum location of pressure measurments around a wing as a dynamic control input in smooth and turbulent conditions, $28^{\text {th }}$ International Congress of the Aeronautical Sciences. (2012)

[9] M. Abdulrahim, H. Garcia, J. Dupuis and R. Lind, Flight Characterictic of Wing Shaping for a Micro Air Vehicle with Membrane Wings, International Forum on Aeroelasticity and Structural Dynamics, IFASD-US-24. (2003)

[10] S. Watkins, M. Abdulrahim, M. Thompson, M. Shortis, B. Loxton, R. Segal, C. Bil and J. Watmuff, An Overview of Experiments on the Dynamic Sensitivity of MAVs to Turbulence, AIAA Guidance, Navigation and Control Conference 2009, Chicago, Illinois, USA, 2009. DOI: $10.2514 / 6.2009-5906$

[11] H. Berge and H. Tijdemen, Theoretical and experimental results for the dynamic response of pressure measuring systems, NLR, Amsterdam, Netherlands, 1965

[12] A. Fisher, S. Watkins and J. Watmuff, Dynamic calibration of pressure measurement system: an improved method, $18^{\text {th }}$ Australasian Fluid Mechanics Conference, Launceston, Australia. (2012)

[13] S. Ravi, The influence of turbulence on a flat plate airfoil at Reynolds numbers relevant to MAVs, School of Aerospace, Mechanical and Manufacturing Engineering, PhD Thesis, RMIT University, Melbourne, Australia, 2011.

[14] G. Vino, An experimental investigation into the time-averaged and unsteady aerodynamics of the simplified passenger vehicle in isolation and in convoys, $\mathrm{PhD}$ thesis, RMIT University. (2005)

[15] M. Marino, Unsteady pressure sensing on a MAV wing for control inputs in turbulence, $\mathrm{PhD}$ Thesis, RMIT University, Melbourne, Australia, 2013.

[16] S.J. Orfanidis, Introduction to signal processing, Prentice Hall Signal Processing Series, Prentice Hall, Upper Saddle River, NJ, USA. (1996)

[17] M. Thompson, S. Watkins and C. White, Span-wise wind fluctuationsin open terrain as applicable to small flying craft, The Aeronautical Journal, 115 (1173). (2011)

[18] M. Marino, S. Watkins and R. Sabatini, Unsteady Pressure Measurements on a MAV Wing for the Design of a Turbulence Mitigation System, IEEE Metrology for Aerospace Conference, Benevento, Italy. (2014) 